\title{
INFORMATION AND USER: SOCIAL MEDIA LITERACY IN DIGITAL SOCIETIES
}

\author{
Dr. Nithin Kalorth \\ Bennett University, Greater Noida \\ Prof. Manish Verma \\ Amity University, Haryana \\ Malvika Sagar \\ Amity University, Greater Noida
}

\begin{abstract}
The information flow in digital societies is discussed and analysed from more a decade with close watch on social media networks. The shift from traditional forms of communication to social media enables users to gratify their daily needs of information digitally. The current paper builds on narrative analysis of selected social media active users and their digital social engagement to understand how a user and a network of user engage with information. To understand role of social media literacy, the current paper interviews the users and correlated the findings with contemporary literature on social media. The results show that social media literacy becomes pillar of information system, but it works in micro-level of societies at crossroads of online and offline spaces.
\end{abstract}

Keywords: Social Media, Active User, Information, Misinformation, Digital Literacy

\section{INTRODUCTION}

The globalization has made people more connected with each other by advancement of technology. The technology in not only bridging the gaps but also brining individuals and societies closer. The identity, power and communication changed its sphere in digital societies. 1970s saw various revolutionary developments in technological, economic, political, and cultural exchanges which lead to globalization. The fast connection and interconnectedness between and among social orders made by the globalization left Information and Communication Technologies (ICTs) to get predominant in each part of social framework where technologies of information handling and communication turned into the epicentre of productivity.

The emergence and popularity of social media networks can be attributed to the active interaction between networked digital societies. The seminal definition of networked societies by Castells (2004) states that "'a society whose social structure is made up of networks powered by micro-electronics-based information and communications technologies". Along with the technological advancements, Castells recognises other factors of new social structure like industrial restructuring for open market and freedom- oriented cultural movements such civil right movements, environmental movement, and feminist movements of 1970s.

Today, we are living in social media reality where, the communication and exitance of an individual and society is marked through social media networks. The information about self, and the societies are created, curated, and distributed through such networks. The process of seeking and providing information is occurring in such networks where the meaning and purpose of information defines the communication. Often, the information created and distributed from a user-to-user level are independent of authoritative and editorial review or consideration. The opinion, belief and personal choices becomes the pillars of information and it takes turns in authenticity. The information in social media often viewed through the lenses of scepticism because of the presence of misinformation, mal-information, and disinformation which are often regular part of a post-truth society (Corner, 2017; Rochlin, 2017; Waisbord, 2018).

The role of social media from is now emerged to a daily life toolkit manifesto from an entertainment and networking platform. The features such as hashtag (\#), geotag, personalised updates provide users the 
information which they are seeking. In other words, the role of mainstream media in informing people about its surrounding is taken over by social media handles (Pentina and Tarafdar, 2014). These handles include official authority, media houses, social influencers, subject experts and common citizen or user. Regardless of the source of the information, it is the individual user who disseminate the information in the larger society through their networks. For example, an information on event (in form of weblink) is posted by a source on Facebook. Now a User A can open the link and can share it on his own Facebook wall, send to different groups on WhatsApp, tweet, or any other social media platform. This network will continue to and endless loops and nodes.

The impact of such information in digital societies are particularly critical as we are going through a crisis in unadulterated information. There are reported cases of fake news leading to pollute the information loop of digital society. Such pollutant information has proved hazardous to take lives of people, manipulate an individual, influencing elections and online scams. India is now becoming an important site for social media platforms. We witnessed immense exclusive India centric marketing campaign by all major social media platforms. The industry experts are also positive about the growing numbers of social media users in India for last few years and social media analyst Keelery assures the growth (Figure 1).

The articulation of such information on social media is difficult to evaluate because of its vast and high-volume big data. In addition, the bulk of real time and historical data of user search and result add complexity in studying the information flow in digital societies. A micro level narrative analysis of the selected users or members of digital society will help us in evaluating the role social media literacy of a user to assess, process and transfer the information.

\section{RESEARCH DESIGN}

The current paper aims to understand how the social media literacy shapes the information consumption, reproduction, and flow in the digital societies. The current studied selected active social media users $(\mathrm{n}=32)$ by conducting in-depth interviews on their social media usage, habits with regard of seeking and disseminating information. The selected users are active in majority of social media, but we selected their responses representing their active interaction on a particular platform (Chart 1). The questions and discussions were asked based on their social media activity observation to understand the role of social media literacy in information dispensing. The proceeding and results of the study is correlated within theoretical context social media and digital society.

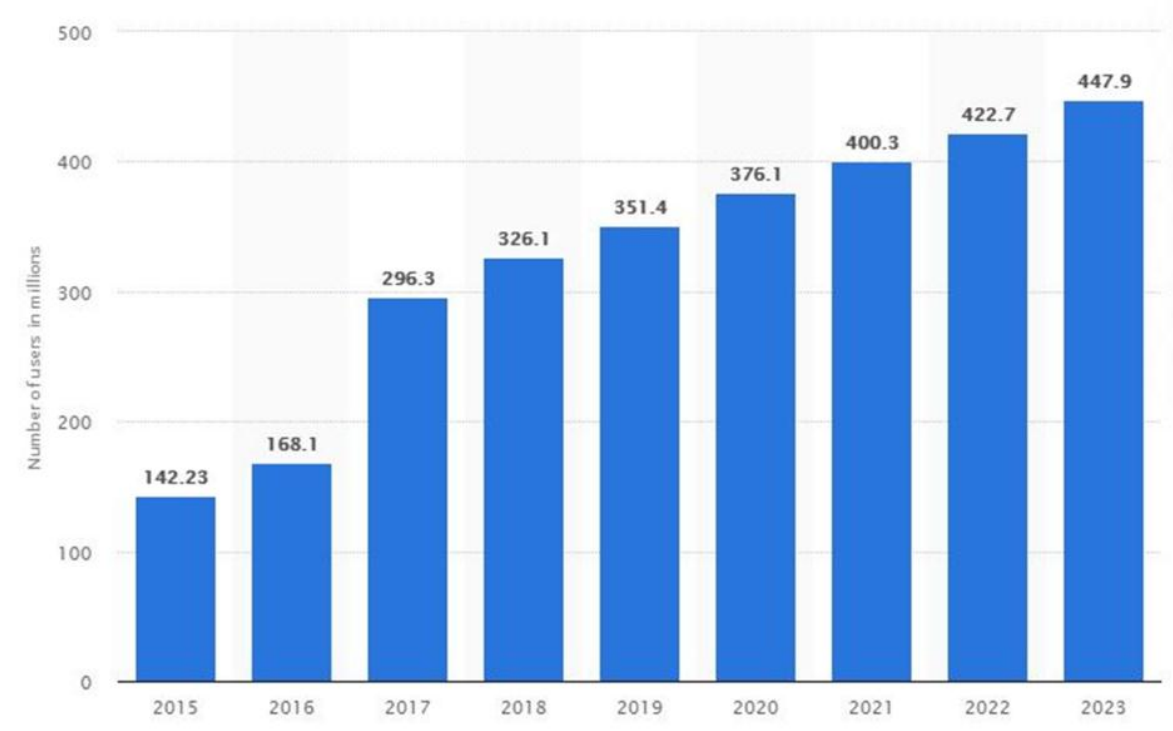

Figure 1: Number of social network users in India from 2015 to 2018 with a forecast until 2023 (in millions) (Keelery, 2020) 
The calculation and determination of active users of social media has been a difficult task for digital ethnographers and social media organizations. In the current study, the selected users are determined to be active by the way the engage in their digital community in terms of contribution to the digital society by creating and disseminating the information. The users from residing in India are from a diverse economic, demographic, and socio- period of 30 years, Thomson (2010) noted that "average sample size of 45 individuals and none of these explicitly reported whether their sample size sought and/or attained saturation". The similar findings are demonstrated in studies conducted by Baker \& Edwards (2012), and Saunders \&Townsend (2016). It will be difficult to call our selected users as social media influencers in terms social media marketing but their contribution

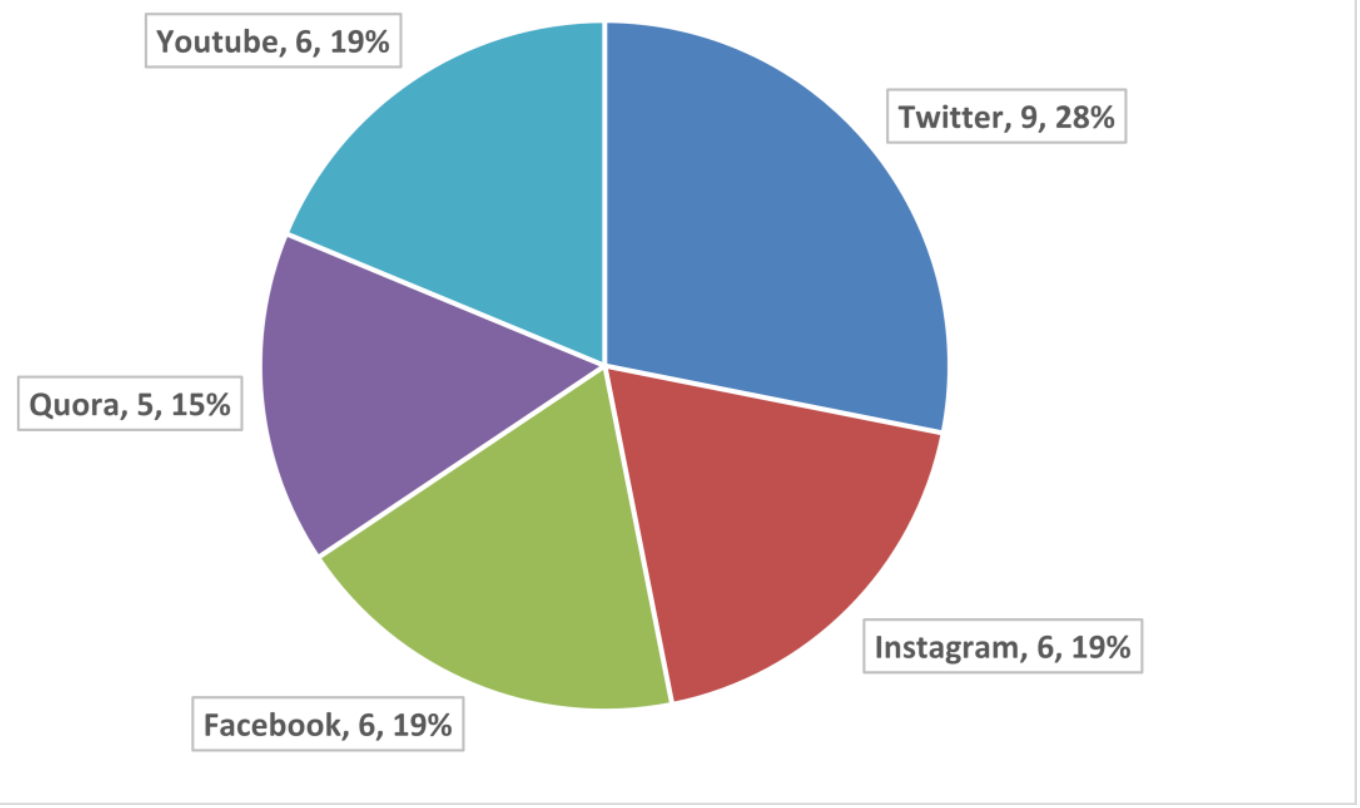

Chart 1: Social Media Platform Distribution of Selected Users

political background. The anonymity of the users is kept for the ethical considerations as the responses they share are noted with the indication as User 1, User 2, User 3 etc. The users were selected from different social media platforms such as Facebook, Twitter, Quora, LinkedIn and YouTube. During the initial stage of current research, there were more than 100 users were selected for observation to understand their engagement in social media. These include the frequency of the posting original content, sharing the existing content, participating in forms of comment and forum discussion and quantity of followers. Later, we approached 60 users for the purpose of the study, from them 32 users agreed to corporate with our project. In a qualitative study, like the current paper, the size of samples is decided based on the nature of the questions and research problem. The volume of sample is often overtaking by the research input from the sample selected. For an instance, to study historical pattern of qualitative research over and engagement on social media are impactful among the digital society. The results of the interview are used in upcoming sections of the paper which divided into digital society, social media user, information and misinformation and social media literacy. When necessary, we have used the direct quote from the users, otherwise, we have taken the essence of their digital experience. The prime aim of this paper is to understand the role of social media literacy for a user to deal with the information around him or her. The understanding of the social media literacy among the selected users are derived from the digital ethnographic ideas of understanding experience, practice, things, social world, relationships, localities, and events (Pink, 2016).

\section{DIGITAL SOCIETY}

Paul and Aithal (2019) understands "digital Society as an interdisciplinary research area and a kind of progressive society that has been formed as a result of adaptation as well as 
integration of advanced technologies into the society and culture". They further identify society, technologies, and content as the stakeholders of digital societies. The user in digital society understands himself as a member of this information society where the information about his environment is received through digitally controlled networks. The traditional ways of information consumption and dissemination are challenged in digital societies where users depend on devices and platforms rather than another person or traditional medium (Ruppert et al, 2013; Montag \& Diefenbach, 2018). Kalorth and Verma (2018) notices the lack of trust in mass media and shift in economic and social life as other reasons for the shift from traditional media to social media by user. Their role of a passive audience is now shifted to an active or engaged user. This shift can be considered as a crucial node in digital societies (Miller, 2020). Earlier, in a traditional society, people used to get an information, test, and verify with their knowledge and experience regardless of the nature of the information. But in digital society, users become the central point of information.

\begin{abstract}
"We can watch live video of an event or any happening on social media platform at our convenience. We can share our response and opinion as comment. This is directly transferred to the sender, mediators of the event - this makes user more empowered and bring him or her to an important phase" (User 3, Political YouTuber).
\end{abstract}

The opinion formed here also act importantly in form of digital public sphere which is technologically connected and impulsive. The public sphere in Indian online spaces were prominently visible in Indian social and political sphere after 2012 Delhi Gang rape. The narratives and counter narratives on social media were led by individual users without gatekeeping. The network of actors in form of opinion leaders trigger the change and through their networks of influences the change in society happens. The entry of technology - to enhance, digital technology, powered a shift in public sphere (Tufekci, 2017, p. 5). When the technological advancement was an obligatory in globalized world, the concept of public sphere in internet or digital world challenged or seemed for a revision on face-to-face community public sphere.

"It is easy to reach people to coordinate. During the initial days of COVID-19 pandemic, there lots of confusion among the public. It was very difficult to address their doubts. But what we saw after that a formation of online teams using various platforms notably WhatsApp and Telegram which includes medical experts, police, social workers, local administration, and citizen. The messages were sent through these channels and it found effective. The pace in which the message reached were amazing. The complexities of offline societies were overcoming by these digital networks which helped us to address the mass." (User 23, also a social activist, uses Quora).

The above said online team or group can be considered as digital public sphere which is different from online. Fraser (1990) views the traditional public sphere as non-uniform network where different group of people comes from different circumstances. Tufekci (2017) calls digital network public sphere as a "complex interaction of publics, online and offline, all intertwined, multiple, connected, and complex, but also transnational and global". Tufekci finds the complexity in digitally networked movements as she terms it as online, informal, and leaderless. But when it comes to an individual user, he gratifies with the information he needs if he bothers about the information. The users interviewed in this current project agreed at various levels that, they are bothered about the information reaching to them. It was also clear that the offline interactions also affect the way they deal with information they engage online. Such results show the prominence of human face-to-face interaction in digital societies where the validation and experimentation of the information occurs. But these can be studies only by uncovering individual users' offline lives. "The way I interact here (Instagram) is very different from my real life. I provide various information in form of home remedies and beauty tips online which often disapproved by mother at home. At home, I feel I am a different girl" (User 11, fashion blogger and Instagrammer). The similar narratives were found from other users shows that the offline spaces are still pertinent, and a close 
encounter is required to uncover the larger depiction of information flow.

\section{Between Information and Misinformation}

"It is not easy to understand who is lying.", said User 6 who is also a $11^{\text {th }}$ grade school student who is active on Instagram by engaging his followers with latest updates on science and technology. He understands the information as a crucial element of daily life. Allcott et al, (2019) studied trends of misinformation on social media to understand the role of platform in combating and controlling the misinformation.

\begin{abstract}
"As a rule, I think no social media stops us from lying. I can tweet that I am in Delhi while I am in Mumbai. From personal point of view, it may be harmless, but the tip of every spiral of lies can be from such personal comment and opinion. Sometimes, we are at a space where information can be too malunions to accept but more danger to left alone" (User 18, Tweets on Gender related issues)
\end{abstract}

It also evident during the current study, that users were confused to determine the validity of the information. This happens when information transfers in rapid way. The selected users which we encountered with raw information often waits for additional reliability and often depends the fact checking sources for validation. This shows the role of fact checkers who work under organization and independent must play a huge in social media literacy. Even though, they do not address the larger society, but they set the tone for the information flow. Krause et al, (2020) suggests that fact-checkers should build trust instead of just saying or implying they are trustworthy like working with actors that are trusted among most of the public.

There are cases of misinformation due to lack of data literacy among journalist, social media managers and influencers (Ireton and Posetti, 2018; Waisbord, 2018). This is more perilous as Lewandowsky et al, (2017) sees that fake news and post-truth will lead to the "decline in social capital, growing economic inequality, increased polarization, declining trust in science, and an increasingly fractionated media landscape". The social media platforms such as Google and Facebook established their fact checking networks through media organizations and educational institutes. Their aim is to make user aware about the information and verify them.

However, it is difficult to uncover the grey area between information and misinformation. In specific, the current paper stands in that grey area to understand how information shaping the digital societies and its actors and networks. "There is an always a space of clarification in offline communication, but online its limited, once said (posted), it said." (User 19, handling social media page of a regional political party). Through the narratives of selected users, it is understood that the public sentiment and its expected outcome reversely affecting the information. The senders of the information foresee the audience reaction which often end up in sending out the "convenient" messages (as quoted by User 1, media teacher and fact checking trainer) which often is fabricated. "Sometimes, it is more important to understand what my followers like to see that what they should see. When I edit videos, I purposefully add some elements to increase attention. But I don't think it is a fake news or misinformation." (User 15, young YouTuber). In the previous comment, the video surfaced of an actor commenting on recently much controversy drug dealing in Bollywood. The Youtuber mixed the video of the actor along with dialogue and shots from the films which she acted. Briefly, it was harmless. But when that 12-minute video from his YouTube channel for edited by someone and surfaced as video stories on WhatsApp and Instagram, it became a new meaning with false intention. The reedited video became more viral than his original video. When asked about this chain of events, he said "It is not in my hands". Here the user feels himself in comfort zone as he is not bothered with the aftereffects of the video. Later, he deleted the original video and posted a clarification. This came after advice with warning from his friends and family on possible damage in future.

\section{Social Media Literacy}

The digital literacy mission in India were controlled by various ICT programs by government. The implementation of ICT in curriculum, conducting awareness programs in rural area and exploring the digital opportunities were some efforts taken by state and central government of India. The contribution of private and NGO bodies in the effort are notable such as consumer awareness 
on online fraud and safe online banking. The user-oriented programs like Digital India Initiative aims to fill the gap between digital services and public so that they can reach the government for needs and explore the opportunities in digital society. The reach and effectiveness of such programs are outside scope of this study, but it was clear from the narratives of selected users that, the social media awareness and literacy were far from the agenda of such campaigns.

\section{"I had chance to interact with rural social media users who ever exposed to social media after entry of high-speed internet services like Jio and low-cost smartphone. They were initially not introduced to any ICT or computer program. They are still afraid to use UPI on mobile, do transaction on internet or even some one never trusts ATM transactions. But they are more confident using Facebook and WhatsApp and reason. They get information from there which bothers them." (User 22, social worker).}

This revelation from the user opens about a bigger picture of social media literacy in India. Here the individual user is engaged more with his or her social media subgroups such as private chat, WhatsApp group, Facebook community group for communication purposes. Varis (2016) noted that online environments studied cannot be taken as selfexplanatory contexts but need to be investigated for locally specific meanings and appropriations. This is to extend the views of Dijck (2013) that the shape of any platform does not determine the way in which people will use it for their communicative purposes, the design of the site will influence interactions. Defaults in digital environments are not just technical but also ideological manoeuvrings which are formed by algorithms, protocols and defaults profoundly shape the cultural experiences of people active on social media platforms. A common user often finds it difficult to break this technical environment but can easily enters to the information zone to retrieve the messages which he or she bothered.

"I flag or report the content which I feel is wrong. More than my feelings, I depend on online verification. The social media platforms provide the option to lie, tell truth and to report the lies and misinformation. But it may not the case with a user who is not bothered to identify or reflect the reality." (User 31. Social media analyst).

Such ignorance from an individual user extends the larger spiral of misinformation and unwillingly contribute to the network of falsehoods. It is also needing to further articulate that the social media users and their digital societies can be understood by analysing their detail encounter with information. The language, structure, and disclosures in social media groups (Facebook, Twitter, etc.) and sub-groups (Facebook group, Facebook pages, etc.) are key variables in process of understanding the concept of social media literacy. The efficiency of the social media networks in determining how digital societies use information rests on aspects to the media firm as well as on the political economy of the radically changed media landscape. The information disseminated by authority like government and peer reviewed articles on mainstream media also surfaces on social media. The individual user often comes in uncertainty to determine the information when the platform focuses on the structure of content rather the content and its authenticity itself. The efforts taken by social media platforms to impart social media literacy is well noted but it should be articulated along with the digital literacy of the user. The policy makers for social media literacy should prepare the content not only for the users who encounter the misinformation from social and political level. They should also focus on the users who deal the social media information and apply those in their day-to-day life. As discussed, the information becomes daily life toolkit for them. The social media literacy programs and initiatives should focus on micro-level points of society rather than overall digital societies.

\section{REFERENCE}

Allcott, H., Gentzkow, M., \& Yu, C. (2019). Trends in the diffusion of misinformation on social media. Research \& Politics, 6(2), 2053168019848554.

Baker, S. E., \& Edwards, R. (2012). How many qualitative interviews is enough? Expert voices and early career reflections on sampling and cases in qualitative 
research. (National Centre for Research Methods Reviews) Southampton, GB.

Castells, M. (2004). Informationalism, networks, and the network society: a theoretical blueprint. The network society: A cross-cultural perspective, 3-45.

Corner, J. (2017). Fake news, post-truth and media-political change. Media, Culture and Society. 39 (7). 1100-1107.

Fraser, N. (1990). Rethinking the public sphere: A contribution to the critique of actually existing democracy. Social text, (25/26), 56-80.

Ireton, C., \& Posetti, J. (2018). Journalism, fake news \& disinformation: handbook for journalism education and training. UNESCO Publishing.

Kalorth, N \& Verma, M (2018). Anatomy of Fake News: On (Mis)information and Belief in the Age of Social Media, Journal of Content, Community \& Communication. 8 (4), 9-14.

Keelery, S (2020). Number of social network users in India from 2015 to 2018 with a forecast until 2023(in millions). Statia. Retrieved on 23 December 2020 from https://www.statista.com/statistics/278 407/number-of-social-network-users-inindia/

Krause, N. M., Freiling, I., Beets, B., \& Brossard, D. (2020). Fact-checking as risk communication: the multi-layered risk of misinformation in times of COVID19. Journal of Risk Research, 1-8.

Lewandowsky, S., Ecker, U. K., \& Cook, J. (2017). Beyond misinformation: Understanding and coping with the "post-truth" era. Journal of applied research in memory and cognition, 6(4), 353-369.

Miller, V. (2020). Understanding digital culture. SAGE Publication.

Montag, C., \& Diefenbach, S. (2018). Towards homo digitalis: important research issues for psychology and the neurosciences at the dawn of the internet of things and the digital society. Sustainability, 10(2), 415.
Paul, P., \& Aithal, P. S. (2018, December). Digital Society: Its Foundation and Towards an Interdisciplinary Field. In Proceedings of National Conference on Advances in Information Technology, Management, Social Sciences and Education (pp. 1-6).

Pentina, I., \& Tarafdar, M. (2014). From "information" to "knowing": Exploring the role of social media in contemporary news consumption. Computers in Human Behavior, 35, 211-223.

Pink, S. (2016). Digital ethnography. Innovative methods in media and communication research, 161-165.

Rochlin, N. (2017). Fake news: belief in posttruth. Library hi tech, 35 (3), 386-392.

Ruppert, E., Law, J., \& Savage, M. (2013). Reassembling social science methods: The challenge of digital devices. Theory, culture $\mathcal{E}$ society, 30(4), 22-46.

Saunders, M. N., \& Townsend, K. (2016). Reporting and justifying the number of interview participants in organization and workplace research. British Journal of Management, 27(4), 836-852.

Thomson, S. B. (2010). Sample size and grounded theory. Thomson, SB (2010). Grounded Theory-Sample Size. Journal of Administration and Governance, 5(1), 45-52.

Tufekci, Z. (2017). Twitter and tear gas: The power and fragility of networked protest. Yale University Press.

Van Dijck, J. (2013). The culture of connectivity: A critical history of social media. Oxford University Press.

Varis, P. (2016). Digital ethnography. The Routledge handbook of language and digital communication, 55-68.

Waisbord, S. (2018). Truth is what happens to news: On journalism, fake news, and post-truth. Journalism studies, 19(13), 18661878. 